\title{
Bedside Medicine: - A Lost Art in High Tech Medicine
}

\section{Opinion}

A doctor's bedside manner is key to the delivery of highquality patient care. Patients are often fearful and anxious. It is the doctor's job to calm their fears and offer hope. A physician's manners and conduct are vital elements of patient care, a tradition that has been passed down from ancient shamans to the rise of modern medicine. Since the $4^{\text {th }}$ century BC, the Greeks were very specific about physician bedside manners. Hippocrates expressed that a physician must be "confidential, very chaste, sober, not a winebibber, and he ought to be fastidious in everything...he should approach the patient with moderate steps...gazing calmly at the sick bed..." [1].

\section{The tradition of bedside medicine in ayurveda}

Ayurveda views medicine as an art of healing where the physician-patient relationship matters the most. The Ayurvedic healing arts address the growing need of compassionate bedside medicine. The ancient Ayurvedic text, Charaka Samhita, says, "A physician, even if well versed in the knowledge of diseases and their treatments, doesn't try to enter into the heart of the patient by virtue of the light of their knowledge, they will not be able to properly heal the disease." Besides knowledge of Ayurveda, a good Ayurvedic practitioner pays attention to physical appearance, body language, demeanor, observation skills, and above all, communication. I like to listen to the concerns of my patients and offer assurance to them with honesty. I do not like to give false hope, but I try to use positive language to help my patients on their journey of healing with compassion and empathy.

Over several centuries, America has seen many innovations in the medical field. Prior to the Civil War, some of the medical care in America included homeopathy, hydrotherapy, electric medicine, and botanical healing. As we developed better techniques for diagnosis and improved therapies for treatments, the practice of medicine began to evolve. Unfortunately, some of the essential bedside manners that were present at early times are disappearing in our technology-focused medical practice. Until the $19^{\text {th }}$ century, the character and behavior of the physician persuaded patients to have faith in the medical professional. However, now good science has become the primary prerequisite. While good behavior is still important, the emphasis of science has risen above empathy. The idea of medicine being an art has faded, and bedside medicine began to slide down [1].

Proper bedside manner involves consoling the patient with support and compassion while remaining honest about a diagnosis. Elements such as a physician's body language, openness, presence, integrity, vocal tone, and attitude all affect bedside manner. When a doctor's bedside manner is bad, a patient may feel scared, dissatisfied, worried, and alone. Therefore, in a difficult diagnosis, a physician has the challenge to not only explain the diagnosis, but also keep the patient comfortable.

A doctor's ability to care for a patient must exemplify an appropriate bedside manner. A physician must connect with the

Opinion
Volume 9 Issue 2 - 2017
Virender Sodhi*
Ayurvedic and Naturopathic medical Clinic, USA
*Corresponding author: Virender Sodhi, Ayurvedic and
Naturopathic medical Clinic, 2115 112th, Ave NE \#4,
Bellevue, WA 98004, USA, Email: virender@Ayush.com
Received: October 22, 2017 | Published: October 24, 2017

patient. A good bedside manner improves communication and reduces errors. Doctors must listen to their patients and utilize the information in their medical treatment. Doctors must build trust and engage the patient. Here are some examples of bedside manners:

a. Making eye contact with the patient.

b. Giving a proper introduction to the patient and family.

c. Using vocabulary that the patient can easily understand.

d. Using honest body language.

e. Listening to the patient carefully with undivided attention

\section{Cancer and bedside medicine}

Bedside behavior is a critical aspect of treating a cancer patient. A friendly, therapeutic relationship between a doctor and a cancer patient has many healing properties. In over three decades of my clinical practice with cancer patients, I know that communication skills are key to providing high-quality care. Oncologists often have the challenge of explaining difficult circumstances clearly to their patients. They do not usually take into consideration the humanity behind their words. Are their words compassionate and empathetic, or are they uncaring behind the patient's back? What is their attitude like? An article published in an oncology journal outlines what oncologists must NOT say to their cancer [2];

A. Physician should not tell cancer patients that I have bad news for them. They have already received bad news. They would not be in my office if they hadn't heard the bad news. As a physician, I cannot act as if I am featuring myself in a soap opera. This only weakens the patient and doctor bond. Being overemotional when conveying information about relapse or progression is not a good idea.

B. Physician should not ask cancer patient if there is a reason he or she did not see a doctor when the symptoms first started. It is unkind to make the patient feel guilty and embarrassed by asking a question to which you already know the answer. As a physician, it might be a matter of deep concern to me to face a patient who ignored their cancer until it might be too late. 
I should not unnecessarily upset the patient by asking such questions. It is a classic passive-aggressive behavior where the patient might derive the feeling that the doctor is deriving pleasure from the patient's misfortune.

C. Oncologist should not tell patients that they are wasting money by taking supplements. Cancer patients want to feel better. Many of them believe that supplements will help their treatment. They feel good to play an active role in their care. Yes, doctors must speak up and stop their patients from taking something injurious! However, if not harmful, as a doctor, I would encourage my cancer patients to take supplements.

D. Physician should not tell patients not to leave their vicinity. The main goal of treating cancer patients is to improve their quality of life. They ought to be able to travel to weddings, graduations, reunions, and so on. In modern-day life, it is safer for patients on chemotherapy to travel out of town. Yes, as a doctor, I should be ready to help out if problems come, but it is not right for me to keep patients from seeing their loved ones in distant areas. You have to be an optimist and believe that everything will be well.

E. Physician should not tell patient that if he or she had not smoked, he or she would not have cancer. If a smoker is sitting in front of a doctor in a medical office, it is immature of the doctor to believe that the smoker with cancer does not know why he or she is sitting in front of the doctor. In other words, you will not help the whole healing process by filling the patient with regret. You can discuss the science behind tobacco-related tumors, particularly if that inspires an accompanying family member to quit smoking. Nevertheless, do not portray an attitude of superiority in front of the patient.

F. Oncologist should not tell patient how many months he or she has to live. Your patient might want to know how much time they have left, but the reality is that no one has seen the future. Many times, oncologists tell their patients how much time they have left. Usually patients take doctor's orders so serious that they die within that time period. Do not be a foolish doctor in pronouncing a patient dead while he or she is alive.

G. An oncologist should not tell the patient that his or her treatment will not make the patient sick. Often times, even with modern supportive care, many patients do have worsening side effects. For instance, many patients lose their hair from chemotherapy. Let them know that there are exceptions to every rule, but it is a good idea to leave absolute guarantees to the all-knowing supreme power.

Cancer care requires addressing a patient's emotions. A 2007 study found that if doctors were better trained to understand the emotional concerns of a patient battling with cancer, the patient's quality of life would be significantly enhanced. When a patient expresses negative emotions, it is the doctor's job to continue the conversation to soothe the anxiety of the patient and respond with empathy. The study demonstrated that when patients expressed negative emotions of fear and worry, the doctors did not always respond with compassion. The scientists recorded 398 clinic conversations between 51 oncologists and 270 patients with advanced cancer, and the oncologists completed surveys. The conversations were coded for the presence of opportunities for oncologists to show empathy to their patients and their actual responses. In 398 conversations, 37\% had at least one empathic opportunity. The range of empathetic opportunities was 0 to 10 , and there were 292 chances to express empathy. The oncologists continued the conversation with empathy $22 \%$ of the time. Female oncologists were more empathetic than male oncologists. Socioemotional oncologists rather than technical oncologists were more likely to respond empathically. The researchers reached a conclusion that the oncologists should respond to their cancer patients with empathy more frequently [3].

I believe that oncologists should be educated to properly respond to the concerns of their patients in a positive manner. With the diagnosis of cancer, the doctor-patient relationship changes to something more than just a medical discussion:

a. The doctor has to educate the patients and answer any of their questions.

b. The doctor must gain an understanding of what the patients know.

c. The doctor should reconcile what the patients know or believe about their condition with the knowledge he has to bring to the patients regarding a balanced understanding of their cancer. As treatment begins, proper bedside manner must be applied.

d. Every patient deals with his or her cancer differently, so an open, personalized, and caring bedside manner must be used.

The empathetic behavior of an oncologist towards his or her cancer patients soothes the anxiety of patients. It has important psychological and physiological health benefits. A good oncologist will not just make a proper diagnosis, but will also communicate that the feelings of the patient are important. An oncologist must listen to his or her patients and honor their voice. Studies show that a positive interaction between a doctor and a cancer patient improves the quality of life of the patient.

\section{Placebo and nocebo medicine}

According to Hippocrates, the mind has a powerful effect on disease and faith, and a firm doctor-patient relationship alongside a treatment plan can lead to significant therapeutic results. A doctor should always aim to stimulate self-healing, known as placebo medicine, to enhance a patient's potential for healing. In Latin, the word placebo means "I shall please." Placebo effect is the process of a doctor using self-healing methods to heal a patient [4].

Mind has a big potential in influencing human health. A placebo response signifies the power of the mind through intention to produce all of the following: a change in oneself, a change in those around one, and a change in the environment in which one lives. Placebo medicine is so powerful, because we have an 
innate capacity for self-healing. However, a patient's belief system provokes self-healing powers of the mind. In today's world, a doctor might be able to diagnose the condition of a patient using modern medical technology. Nevertheless, if the diagnosis, therapy, and therapeutic interaction between doctor and patient do not encourage the hope and faith in a patient, the chances of success in healing decrease tremendously. In other words, the attitudes of the patient and the doctor, as well as their faith in each other and the process, are very important in producing therapeutic results [5].

The placebo effect is a direct effect of a doctor's ability to heal and cure the patient seeking help. If the belief and expectation of the patient and the doctor as well as the interaction between the doctor and the patient are harmonious, the placebo effect is enhanced. Although nothing is being transmitted from the healer to the patient, the faith of the patient in the doctor is very helpful in healing the patient. Research shows that the patient and the doctor's mindset even change the physiology in the patient. When a doctor shows empathy and confidence to a patient, brain chemicals change in the patient's brain. As a patient feels cared about by the doctor, it lowers the secretion of stress hormones. A healthy doctor-patient relationship creates less anxiety in patients and more relaxation [5].

As an authority on a patient's health, a doctor can create a positive or a negative effect (placebo effect or nocebo effect) on a patient's wellbeing. A nocebo effect occurs as a result of placebo therapy creating negative changes in the path of treatment. Placebo and nocebo effects are psychobiological phenomena They take rise from various factors: nature of treatment, patient treatment expectations and experience, verbal and non-verbal communications by the doctor, as well as the patient-doctor interaction. The verbal and nonverbal communications of doctors that may unconsciously contain negative recommendations may lead to a nocebo response biologically in a patient [4].
Many conventional doctors do not understand the science of natural self-healing. In naturopathic medicine, placebo medicine is very real, and it plays a critical role in healing a patient. There is nothing wrong with adding placebo effect for better healing. It is free and does not have any side effects. However, doctors have a huge responsibility in this matter. They must speak the truth to their patients regarding risks, but they have to be careful in what they say. Studies show that there are more nocebo effects when patients are given negative expectations. Doctors must frame their words positively when treating a patient, as putting someone in a negative mindset can negatively influence their health and wellbeing.

\section{Acknowledgment}

None.

\section{Conflict of Interest}

None.

\section{References}

1. Silverman BD (2012) Physician behavior and bedside manners: the influence of William Osler and The Johns Hopkins School of Medicine. Proc (Bayl Univ Med Cent) 25(1): 58-61.

2. (2014) 7 things oncologists should never say to their patients. CancerNetwork.

3. Pollak KI, Arnold RM, Jeffreys AS, Alexander SC, Olsen MK, et al (2007) Oncologist communication about emotion during visits with patients with advanced cancer. J Clin Oncol 25(36): 5748-5752.

4. Häuser W, Hansen E, Enck P (2012) Nocebo Phenomena in Medicine: Their Relevance in Everyday Clinical Practice. Dtsch Arztebl Int 109(26): 459-465.

5. Bennett P W (2016) Placebo and the Power to Heal. Musculoskeletal Key. 